\title{
Furthering our understanding of the needs of adolescent children of parents with Parkinson's disease
}

This article was published in the following Dove Press journal:

Adolescent Health, Medicine and Therapeutics

26 July 2013

Number of times this article has been viewed

\author{
David Morley \\ Crispin Jenkinson \\ University of Oxford, Nuffield \\ Department of Population Health, \\ Old Road Campus, Oxford, UK
}

Correspondence: David Morley University of Oxford, Nuffield Department of Population Health, Old Road Campus,

Oxford OX3 7LF, UK

Tel +44018 65289432

Email david.morley@dph.ox.ac.uk
Abstract: A significant body of research has developed in recent years that has aided our understanding of how a range of conditions might affect the offspring of affected individuals. Research relating to the impact of parental Parkinson's disease, however, is still in its relative infancy. This short paper focuses mainly, but not exclusively, on adolescence and briefly outlines findings to date. This is followed by a discussion of research that needs to be undertaken in order to further our understanding of the needs of children of people with Parkinson's.

Keywords: Parkinson's disease, parent, adolescent, children

\section{Introduction}

Research into the potential impact of a variety of parental conditions on children of a range of ages has increased significantly over the last two decades. Some conditions, such as multiple sclerosis, are now relatively well explored and this has allowed for the development of interventions and inclusion of children's needs in clinical guidelines. ${ }^{1,2}$ Research relating to the impact of parental Parkinson's disease (PD), however, is still in its relative infancy, and the importance of furthering our understanding should not be underestimated. Whilst PD is largely associated with the latter stages of adulthood, this is not always so. In approximately $5 \%-10 \%$ of cases, Parkinson's is apparent before the age of 40 years, and one in seven cases is diagnosed before the age of 50 years..$^{3,4}$ Consequently many people with Parkinson's (PwP) are likely to be raising young and adolescent children. Additionally, with an aging population and the subsequent increase in the prevalence of $\mathrm{PD},{ }^{5}$ many older adult children are likely to experience the slow, protracted deterioration that characterizes the condition. The focus of this brief discussion, however, is mainly on young people aged between approximately 11 years and the late teens. These are not "young carers" as classically discussed in the literature. 6,7 Rather, they are young people who are not fulfilling a primary caregiving role but are likely to experience a range of challenges as a consequence of a parent's Parkinson's.

\section{Previous research}

A scoping review of previous research demonstrates the limited nature of research in this area. To date, only a handful of studies have been conducted, although they have identified some key challenges that adolescents might face when confronted with parental PD. The qualitative work reported by Grimshaw ${ }^{8}$ was the first to make a direct assessment of the offspring of PwP. From his work with a small sample of children and young people aged 5-24 years, Grimshaw identified a number of 
potential concerns. These included the emotional and social development of the child, the transition to independence, and the social embarrassment related to parental PD, something particular heightened in adolescents. Also noted was a significant degree of role change within the family unit. Children's emotional and domestic roles were changing as a result of their parent's PD, and subsequent changes in relationships with both the affected and nonaffected parent were evident. Some such issues were later reflected in a short piece written by Rees ${ }^{9}$ who lays bare the impact of having a mother diagnosed with PD at 46 years when she herself was just 10 years of age.

Subsequent quantitative research on children of PwP has identified a number of challenges they may face, including problems with peer relationships, role adjustment within the family, and fears for the future. Such challenges may be related to certain demographic variables, eg, the duration of the parent's PD and/or the age of the young person. ${ }^{10,11}$ The evidence also suggests that some variables related to family structure might influence the young person's response to parental PD. For example, those without the support of brothers and sisters appear to show inferior adjustment to parental illness in comparison with those who have siblings. ${ }^{12}$ Issues concerning the provision of support and sources of relevant information for children of $\mathrm{PwP}$ of all ages are well documented. ${ }^{10,11,13}$ Mental health may also be vulnerable, with adolescent children of PwP approximately twice as likely to be experiencing at least low-level depression in comparison with the normal population. ${ }^{10,13}$ Such data emphasize the need for children experiencing parental illness to be carefully monitored by relevant professionals. ${ }^{14}$

\section{Future research}

Whilst the research outlined above is significant in its importance, there remains much scope for further studies in order to gain a fuller understanding, as noted by others prominent in the field of PD. ${ }^{15}$ Most importantly, longitudinal research is required to examine how the sequelae of Parkinson's affects the offspring of affected individuals over time. Such data are essential for chronic progressive conditions such as PD. A number of the challenges that the young person might encounter, eg, deciding when to leave the parental home or whether to pursue higher education, are likely to become increasingly problematic in light of their affected parent's worsening condition. Longitudinal data could also offer opportunities for developing appropriate interventions, in addition to identifying those families most "at risk".
A further issue to be addressed is the current lack of comparative data from the offspring of healthy parents when assessing those of PwP. The absence of such data means that conclusions on a range of potentially important issues cannot be drawn. Control groups are therefore essential in future research. Additionally, research is required that considers the impact of parental PD on younger children. Research to date has focused almost entirely on adolescent and adult children, ${ }^{10-12}$ and whilst the numbers of children below the age of 11 years will probably be, at best, modest, the impact of parental PD on these younger children could be significant. The development of interventions such as those for young children of parents with multiple sclerosis ${ }^{1}$ and cancer ${ }^{16}$ might be of significant value for younger children of PwP. Interventions such as these allow children to meet with others in a similar situation to themselves and can help in allowing children to articulate and share their concerns in a sympathetic and nonthreatening setting. Such an environment would also be conducive to furnishing younger children with targeted, age-appropriate information that enables them to better understand what is happening to their parent with Parkinson's.

There are still additional groups and demographic variables that need to be addressed if we are to make a comprehensive examination of factors influencing childrens' response to parental PD. The need to examine different cultures has been identified as an important issue in Parkinson's research. ${ }^{8,17}$ With this in mind, if diverse groups are to be investigated, it is essential to acknowledge that alternative cultures could have very different needs. A particular example might be those ethnic groups where significant importance is placed on the social support network of the close and wider family unit. Another potentially important target of interest is single-parent families as no research is currently available on the impact of parental PD in this group. Data has indicated that the prevalence of child and adolescent mental disorder is double that in lone parent families when compared with two parent families. ${ }^{18}$ Given the propensity for children of PwP to be more susceptible to mental health problems, ${ }^{10,11,13}$ those within a single-parent family may be particularly vulnerable. Finally, there may well be further variables specific to parental PD that need to be examined. For example, research might focus on how current therapeutic approaches to PD (ie, deep brain stimulation, levodopa/carbidopa infusion) and the onset of neuropsychiatric symptoms (ie, depression, psychosis) might impact on the well-being of the child. 


\section{Conclusion}

This short piece has attempted to summarize our current knowledge of the impact of parental PD. Additionally, it has identified some shortcomings in research to date and priorities for research in the future. In $2002 \mathrm{Rees}^{9}$ concluded $^{-}$ "I would hope that now medical teams have a better understanding of the difficulties encountered by patients with Parkinson's disease and their families, but I wonder." More than a decade on, we can probably say that we do indeed have a greater understanding. But, do we yet know enough? On the basis of the brief discussion presented here, the answer appears to be "no", although we have made some headway. It is therefore imperative that the momentum developed over the last decade is maintained if we are to continue to further our understanding of the challenges faced by children, of all ages, whose lives are affected by parental PD.

\section{Disclosure}

This paper was prepared without external funding and the authors declare no conflicts of interest.

\section{References}

1. Mutch K. Information for young people when multiple sclerosis enters the family. Br J Nurs. 2005;14:758-767.

2. National Institute for Health and Clinical Excellence. Multiple Sclerosis: National Clinical Guideline for Diagnosis and Management in Primary and Secondary Care. London, UK: National Institute for Health and Clinical Excellence; 2004.

3. Clarke CE, Moore AP. Parkinson's disease. Am Fam Physician. 2007;75: 1045-1048.
4. Koller W, Hubble J. Young-onset Parkinson's disease. In: Johnson A, editor Young Parkinson's Handbook: A Guide for Patients and Their Families. New York: The American Parkinson's Disease Society; 1995.

5. Archibald N, Miller N, Rochester L. Neurorehabilitation in Parkinson's disease. Handb Clin Neurol. 2013;110:435-442.

6. Aldridge J, Becker S. Children as carers. Arch Dis Child. 1993;69: 459-462.

7. Dearden C, Becker S, Aldridge J. Children who care: a case for nursing intervention? Br J Nurs. 1995;4:698-701.

8. Grimshaw R. Children of Parents with Parkinson's Disease. A Research Report for the Parkinson's Disease Society. London, UK: National Children's Bureau; 1991.

9. Rees L. Living with Parkinson's disease - a child's perspective. BMJ 2002;324:1562.

10. Schrag A, Morley D, Quinn N, Jahanshahi M. Impact of Parkinson's disease on patients' adolescent and adult children. Parkinsonism Relat Disord. 2004;10:391-397.

11. Morley D. The psychosocial impact of Parkinson's disease on the wider family unit: a focus on the offspring of affected individuals. In: Rana AQ, editor. Symptoms of Parkinson's Disease. Rijeka, Croatia: InTech Open Access; 2011.

12. Morley D, Selai C, Thompson A. Sibling support and adjustment to parental neurological illness. Qual Life Res. 2010;19(S1):143-144.

13. Morley D, Selai C, Schrag A, Jahanshahi M, Thompson A. Adolescent and adult children of parents with Parkinson's disease: incorporating their needs in clinical guidelines. Parkinsons Dis. 2011;2011:951874.

14. Morley D, Jenkinson C. The importance of recognising depression in adolescents affected by parental illness. Psychology. 2012;3(9A): 756-757.

15. McNamara P. The emotional challenges of children whose parents have Parkinson's disease. Available from: http://parkinsons.about.com/od/ forcaregivers/a/children_of_pd.htm. Accessed January 31, 2013.

16. Greening K. Support groups for children of patients with cancer. Cancer Pract. 1999; 7:208-211.

17. Parkinson's Disease Society. Towards Diversity. London: Parkinson's Disease Society; 2004.

18. Meltzer H, Gatward R, Goodman R, Ford T. Mental health of children and adolescents in Great Britain. Int Rev Psychiatry. 2003;15: 185-187.
Adolescent Health, Medicine and Therapeutics

\section{Publish your work in this journal}

Adolescent Health, Medicine and Therapeutics is an international, peer-reviewed, open access journal focusing on health, pathology, and treatment issues specific to the adolescent age group. All aspects of health maintenance, preventative measures and disease treatmen interventions are addressed within the journal and practitioners from

\section{Dovepress}

all disciplines are invited to submit their work as well as healthcare researchers and patient support groups.. The manuscript management system is completely online and includes a very quick and fair peerreview system. Visit http://www.dovepress.com/testimonials.php to read real quotes from published authors. 